\title{
Greedy Approximation for the Source Location Problem with Vertex-Connectivity Requirements in Undirected Graphs *
}

\author{
Toshimasa Ishii ${ }^{\text {a,* }}$

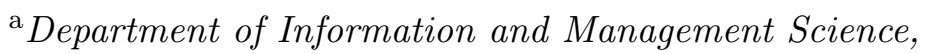 \\ Otaru University of Commerce, \\ Otaru-city Hokkaido 047-8501 Japan
}

\begin{abstract}
Let $G=(V, E)$ be a simple undirected graph with a set $V$ of vertices and a set $E$ of edges. Each vertex $v \in V$ has a demand $d(v) \in Z_{+}$, and a cost $c(v) \in R_{+}$, where $Z_{+}$and $R_{+}$denote the set of nonnegative integers and the set of nonnegative reals, respectively. The source location problem with vertex-connectivity requirements in a given graph $G$ asks to find a set $S$ of vertices minimizing $\sum_{v \in S} c(v)$ such that there are at least $d(v)$ pairwise vertex-disjoint paths from $S$ to $v$ for each vertex $v \in V-S$. It is known that the problem is not approximable within a ratio of $O\left(\ln \sum_{v \in V} d(v)\right)$, unless NP has an $O\left(N^{\log \log N}\right)$-time deterministic algorithm. Also, it is known that even if every vertex has a uniform cost and $d^{*}=4$ holds, then the problem is NP-hard, where $d^{*}=\max \{d(v) \mid v \in V\}$.

In this paper, we consider the problem in the case where every vertex has uniform cost. We propose a simple greedy algorithm for providing a $\max \left\{d^{*}, 2 d^{*}-6\right\}$ approximate solution to the problem in $O\left(\min \left\{d^{*}, \sqrt{|V|}\right\} d^{*}|V|^{2}\right)$ time, while we also show that there exists an instance for which it provides no better than a $\left(d^{*}-1\right)$ approximate solution. Especially, in the case of $d^{*} \leq 4$, we give a tight analysis to show that it achieves an approximation ratio of 3 . We also show the APX-hardness of the problem even restricted to $d^{*} \leq 4$.
\end{abstract}

Key words: graph algorithm, greedy algorithm, undirected graph, location problem, vertex-connectivity 


\section{Introduction}

Problems of selecting the best location of facilities in a given network to satisfy a certain property are called location problems [12]. Recently, the location problems with requirements measured by a network-connectivity have been studied extensively $[2,3,5,7,6,9-11,15-18]$.

Connectivity and/or flow-amount are very important factors in applications to control and design of multimedia networks. In a multimedia network, a set $S$ of some specified network nodes, such as the so-called mirror servers, may have functions of offering the same services for users. A user at a node $v$ can use the service by communicating with at least one node $s \in S$ through a path between $s$ and $v$. The flow-amount (which is the capacity of paths between $S$ and $v$ ) affects the maximum data amount that can be transmitted from $S$ to a user at a node $v$. Also, the edge-connectivity or the vertex-connectivity between $S$ and $v$ measures the robustness of the service against network failures. The concept of such connectivity and/or flow-amount between a node and a set of specified nodes was given by H. Ito [8], considering design of a reliable telephone network with plural switching apparatuses.

Given a graph, the problem of finding the best location of such a set $S$ of vertices, called sources, under connectivity and/or flow-amount requirements from each vertex to $S$ is called the source location problem, which is formulated as follows:

Problem 1 (Source location problem with meausre $\psi$ )

Input : A graph $G=(V, E)$ with a set $V$ of vertices and a set $E$ of edges with nonnegative real capacities, a cost function $c: V \rightarrow R_{+}$(where $R_{+}$denotes the set of nonnegative reals), and a demand function $d: V \rightarrow R_{+}$.

Output : A vertex set $S \subseteq V$ such that $\psi(S, v) \geq d(v)$ holds for every vertex $v \in V-S$ and $\sum_{v \in S} c(v)$ is minimized, where $\psi(S, v)$ is a measure based on the edge-connectivity, vertex-connectivity or flow-amount between $S$ and a vertex $v$ in the input graph $G$.

For such measures $\psi(S, v)$, one may consider the minimum capacity $\lambda(S, v)$ of an edge cut $C \subseteq E$ that separates $v$ from $S$, the minimum size $\kappa(S, v)$ of a vertex cut $C \subseteq V-S-v$ that separates $S$ and $v$, or the maximum number

\footnotetext{
ऋ A preliminary version of this paper was accepted at the 18th International Symposium on Algorithms and Computation (ISAAC'2007), Sendai, Japan, December 2007.

* Corresponding author. Department of Information and Management Science, Otaru University of Commerce, Otaru-city Hokkaido 047-8501 Japan, Tel\&Fax: +81-134-27-5389.

Email address: ishii@res.otaru-uc.ac.jp (Toshimasa Ishii).
} 
$\hat{\kappa}(S, v)$ of paths between $S$ and $v$ such that no pair of paths has a common vertex in $V-v$.

Here let us review the developments in the source location problems in undirected graphs. The problem with $\psi=\lambda$ was first considered by Tamura et al. [17]. They showed that the problem with uniform costs and uniform demands can be solved in polynomial time. Also, Tamura et al. [18] showed that the case of uniform costs and general demands is solvable in polynomial time, while the fastest known algorithm for it achieves complexity $O(m M(n, m))$ due to Arata et al. [2], where $n=|V|, m=|\{\{u, v\} \mid u, v \in V\}|$, and $M(n, m)$ denotes the time for max-flow computation in the graph with $n$ vertices and $m$ edges. In general, Sakashita et al. [16] showed that the problem is strongly NP-hard. It is also known that when a given graph is a tree, the problem is weakly NP-hard [2] and there exists a pseudo-polynomial time algorithm for it $[11,16]$.

For $\psi=\kappa$, Ito et al. [9] investigated the problem with uniform costs and uniform demands $d(v)=k$, presented a polynomial time algorithm in the case of $k \leq 2$, and showed the NP-hardness of the problem in the case of $k \geq 3$. They also showed that in the case of $k \leq 2$, even if a measure $\lambda(S, v) \geq \ell$ is added, then the problem is still solvable in polynomial time.

For $\psi=\hat{\kappa}$, Nagamochi et al. [15] showed that the problem with uniform demands $d(v)=k$ can be solved in $O\left(\min \{k, \sqrt{n}\} k n^{2}\right)$ time. In [7], Ishii et al. considered the problem with uniform costs and general demands, and showed that it can be solved in linear time in the case of $d^{*} \leq 3$, while it is NP-hard even restricted to $d^{*}=4$, where $d^{*}=\max \{d(v) \mid v \in V\}$. They also showed that if $d^{*} \leq 3$, then even in the case of general costs, it is also solvable in polynomial time[6].

Also for directed graphs, many variants of problems have been investigated (see $[3,5,10]$ for $\psi=\lambda$, [15] for $\psi=\hat{\kappa}$, and [14] for a survey).

Recently, Sakashita et al.[16] showed that no problems of the above three types of connectivity requirements in undirected/directed graphs are approximable within the ratio of $O\left(\ln \sum_{v \in V} d(v)\right)$, unless NP has an $O\left(N^{\log \log N}\right)$-time deterministic algorithm. They also gave $\left(1+\ln \sum_{v \in V} d(v)\right)$-approximation algorithms for all such problems if the capacity and demand functions are integral.

In this paper, we focus on the problem with $\psi=\hat{\kappa}$ in undirected graphs. As shown in [16], in general, it is unlikely that it is approximable within a ratio of $O\left(\ln \sum_{v \in V} d(v)\right)$. Moreover, it was shown in [7] that even if the cost function is uniform and $d^{*}$ is bounded from above by a constant, the problem is NP-hard. In this paper, after describing some definitions and preliminaries in Section 2, we show in Section 3 that if the cost function is uniform, then a simple greedy algorithm provides a $\max \left\{d^{*}, 2 d^{*}-6\right\}$-approximate solution in 
$O\left(\min \left\{d^{*}, \sqrt{n}\right\} d^{*} n^{2}\right)$ time; the approximation ratio is constant if $d^{*}$ is bounded from above by a constant. Especially, in Section 4, in the case of $d^{*} \leq 4$, we give a tight analysis to show that it achieves an approximation ratio of 3 . We also show that the problem is APX-hard even restricted to uniform costs and $d^{*} \leq 4$.

Before closing this section, we summarize our method. First, we start with the source set $S=V$. Then, we pick vertices $v$, one by one, in nondecreasing order of their demands; only when $S-\{v\}$ remains feasible, then update $S:=S-\{v\}$. It was shown in [2] that for the problem with $\psi=\lambda$ and uniform costs in undirected graphs, this algorithm provides an optimal solution. In our problem, this method may not achieve an optimal, but an approximation ratio of $\max \left\{d^{*}, 2 d^{*}-6\right\}$.

\section{Main Theorems}

Let $G=(V, E)$ be a simple undirected graph with a set $V$ of vertices and a set $E$ of edges, where we denote $|V|$ by $n$ and $|E|$ by $m$. A singleton set $\{x\}$ may be simply written as $x$, and " $\subset$ " implies proper inclusion while " $\subseteq$ " means " $\subset$ " or "=". The vertex set and edge set of a graph $G$ are denoted by $V(G)$ and $E(G)$, respectively. For a vertex subset $V^{\prime} \subseteq V, G\left[V^{\prime}\right]$ means the subgraph induced by $V^{\prime}$. For a vertex set $X \subseteq V, N_{G}(X)$ is defined as the set of all vertices in $V-X$ which are adjacent to some of vertices in $X$. Moreover, let $N_{G}(\emptyset)=\emptyset$. For a vertex set $Y \subseteq V$ and a family $\mathcal{X}$ of vertex sets, $Y$ covers $\mathcal{X}$ if each $X \in \mathcal{X}$ satisfies $X \cap Y \neq \emptyset$. For a family $\mathcal{X}$ of vertex sets in $V$, the frequency of a vertex $v$ (with respect to $\mathcal{X}$ ) is defined as the number of sets of $\mathcal{X}$ which includes $v$, and let $f(V, \mathcal{X})$ denote the maximum frequency with respect to $\mathcal{X}$ of a vertex in $V$.

For a vertex $v \in V$ and a vertex set $X \subseteq V-\{v\}$ in $G$, we denote by $\hat{\kappa}_{G}(X, v)$ the maximum number of paths from $v$ to $X$ such that no pair of paths has a common vertex in $V-v$. For a vertex $v \in V$ and a vertex set $X \subseteq V$ with $v \in X$, let $\hat{\kappa}_{G}(X, v)=\infty$. By Menger's theorem, the following lemma holds.

Lemma 2 For a vertex $v \in V$ and a vertex set $X \subseteq V-\{v\}, \hat{\kappa}_{G}(X, v) \geq k$ holds if and only if $\left|N_{G}(W)\right| \geq k$ holds for every vertex set $W \subseteq V-X$ with $v \in W$.

In this paper, each vertex $v \in V$ in $G=(V, E)$ has a nonnegative integer demand $d(v)$. Let $d^{*}=\max \{d(v) \mid v \in V\}$. A vertex set $S \subseteq V$ is called a source set if it satisfies

$$
\hat{\kappa}_{G}(S, v) \geq d(v) \text { for all vertices } v \in V-S,
$$


and we call each vertex $v \in S$ a source. In this paper, we consider the following source location problem with local vertex-connectivity requirements in an undirected graph (shortly, LVSLP or $d^{*}$ LVSLP).

Problem 3 (LVSLP or $\left.d^{*} L V S L P\right)$

Input : An undirected graph $G=(V, E)$ and a demand function $d: V \rightarrow Z_{+}$ (where $Z_{+}$denotes the set of nonnegative integers).

Output : A source set $S \subseteq V$ with the minimum cardinality.

The main results of this paper are described as follows.

Theorem 4 Given an undirected graph $G=(V, E)$ and a demand function $d: V \rightarrow Z^{+}, \operatorname{LVSLP}$ is $\max \left\{d^{*}, 2 d^{*}-6\right\}$-approximable in $O\left(\min \left\{d^{*}, \sqrt{n}\right\} d^{*} n^{2}\right)$ time.

Theorem 5 A 3-approximate solution to $4 L V S L P$ can be found in $O\left(n^{2}\right)$ time, while $4 L V S L P$ is APX-hard.

In the subsequent sections, we will prove these theorems constructively by giving an approximation algorithm for LVSLP. Also, we will show that there exists an instance for which the proposed algorithm provides no better than a $\left(d^{*}-1\right)$-approximate solution.

In the rest of this section, we introduce several properties for LVSLP, which will be used in the subsequent sections. For a vertex set $X \subseteq V, d(X)$ denotes the maximum demand among all vertices in $X$, i.e., $d(X)=\max _{v \in X} d(v)$ (note that we define $\left.\max _{\emptyset}=0\right)$. A vertex subset $W \subseteq V$ with $d(W)>\left|N_{G}(W)\right|$ is called a deficient set. We have the following property by Lemma 2 .

Lemma 6 A vertex set $S \subseteq V$ is a source set if and only if $S$ satisfies $W \cap S \neq$ $\emptyset$ for every deficient set $W$.

A deficient set $W$ is minimal if no proper subset of $W$ is deficient. For a vertex $v \in V$, we say that a deficient set $W \subseteq V$ with $v \in W$ is a minimal deficient set with respect to $v$, if $W$ is minimal deficient and $d(v)>\left|N_{G}(W)\right|$. A minimal deficient set has the following properties.

Lemma 7 [7] Every minimal deficient set $W$ with respect to $v \in W$ induces a connected graph.

Lemma 8 Let $W$ be a minimal deficient set with respect to $v \in W$. If there is a set $X$ with $v \notin X,\left|N_{G}(X) \cap W\right|=1$, and $X \cap N_{G}(W) \neq \emptyset$, then $N_{G}(X) \cap W=\{v\}$.

PROOF. Assume by contradiction that $v \in(W-X)-N_{G}(X)$. Now we have 
$N_{G}\left((W-X)-N_{G}(X)\right) \subseteq\left(N_{G}(W)-X\right) \cup\left(W \cap N_{G}(X)\right)$. Hence, it follows from $\left|N_{G}(W) \cap X\right| \geq 1$ and $\left|W \cap N_{G}(X)\right|=1$ that $\left|N_{G}\left((W-X)-N_{G}(X)\right)\right| \leq$ $\left|N_{G}(W)-X\right|+\left|W \cap N_{G}(X)\right| \leq\left|N_{G}(W)\right|-\left|N_{G}(W) \cap X\right|+1 \leq\left|N_{G}(W)\right|<d(v)$ and $(W-X)-N_{G}(X)$ is also a deficient set, contradicting the minimality of $W$.

For two vertex sets $X$ and $Y$, we say that $X$ and $Y$ intersect each other, if none of $X \cap Y, X-Y$, and $Y-X$ is empty. For two vertex sets $X$ and $Y$, the following holds.

$\left|N_{G}(X)\right|+\left|N_{G}(Y)\right| \geq\left|N_{G}(X \cap Y)\right|+\left|N_{G}(X \cup Y)\right|$.

$\left|N_{G}(X)\right|+\left|N_{G}(Y)\right| \geq\left|N_{G}\left((X-Y)-N_{G}(Y)\right)\right|+\left|N_{G}\left((Y-X)-N_{G}(X)\right)\right|$.

Lemma 9 Let $W_{i}, i=1,2$ be minimal deficient sets with respect to $w_{i} \in W_{i}$. If $W_{1}$ and $W_{2}$ intersect each other, $w_{1} \in W_{1}-W_{2}$, and $w_{2} \in W_{2}-W_{1}$, then $w_{1} \in N_{G}\left(W_{2}\right)$ or $w_{2} \in N_{G}\left(W_{1}\right)$ hold.

PROOF. Assume by contradiction that $\left\{w_{1}, w_{2}\right\} \cap\left(N_{G}\left(W_{1}\right) \cup N_{G}\left(W_{2}\right)\right)=\emptyset$. By $w_{1} \in\left(W_{1}-W_{2}\right)-N_{G}\left(W_{2}\right)$ and $w_{2} \in\left(W_{2}-W_{1}\right)-N_{G}\left(W_{1}\right)$, we have $\left(W_{1}-W_{2}\right)-N_{G}\left(W_{2}\right) \neq \emptyset \neq\left(W_{2}-W_{1}\right)-N_{G}\left(W_{2}\right)$. Now we have $\left|N_{G}\left(W_{1}\right)\right|<$ $d\left(w_{1}\right)$ and $\left|N_{G}\left(W_{2}\right)\right|<d\left(w_{2}\right)$, since $W_{1}$ and $W_{2}$ are both deficient sets. It follows from (3) that we have $d\left(w_{1}\right)>\left|N_{G}\left(\left(W_{1}-W_{2}\right)-N_{G}\left(W_{2}\right)\right)\right|$ or $d\left(w_{2}\right)>$ $\left|N_{G}\left(\left(W_{2}-W_{1}\right)-N_{G}\left(W_{1}\right)\right)\right|$ (say, $\left.d\left(w_{1}\right)>\left|N_{G}\left(\left(W_{1}-W_{2}\right)-N_{G}\left(W_{2}\right)\right)\right|\right)$. Then $\left(W_{1}-W_{2}\right)-N_{G}\left(W_{2}\right)$ is also deficient, which contradicts the minimality of $W_{1}$. Hence, it follows that $\left\{w_{1}, w_{2}\right\} \cap\left(N_{G}\left(W_{1}\right) \cup N_{G}\left(W_{2}\right)\right) \neq \emptyset$.

\section{Greedy Algorithm}

For a given graph $G=(V, E)$ and a demand function $d: V \rightarrow Z_{+}$, let opt $(G, d)$ denote the optimal value to LVSLP. In this section, we give a simple greedy algorithm, named GREEDY_LVSLP, for finding a $\max \left\{d^{*}, 2 d^{*}-6\right\}$-approximate solution $S$ to LVSLP in $O\left(\min \left\{d^{*}, \sqrt{n}\right\} d^{*} n^{2}\right)$ time. Below, assume that the given graph $G$ is connected, since if $G$ is disconnected, then we can consider the problem for each connected component separately.

The algorithm GREEDY_LVSLP is a greedy method to find a minimal feasible solution $S_{0}$. We start with the source set $S_{0}=V$, and pick vertices $v \in V$, one by one, in nondecreasing order of their demands. Only when $S_{0}-\{v\}$ remains to be a source set, we update $S_{0}:=S_{0}-\{v\}$. 


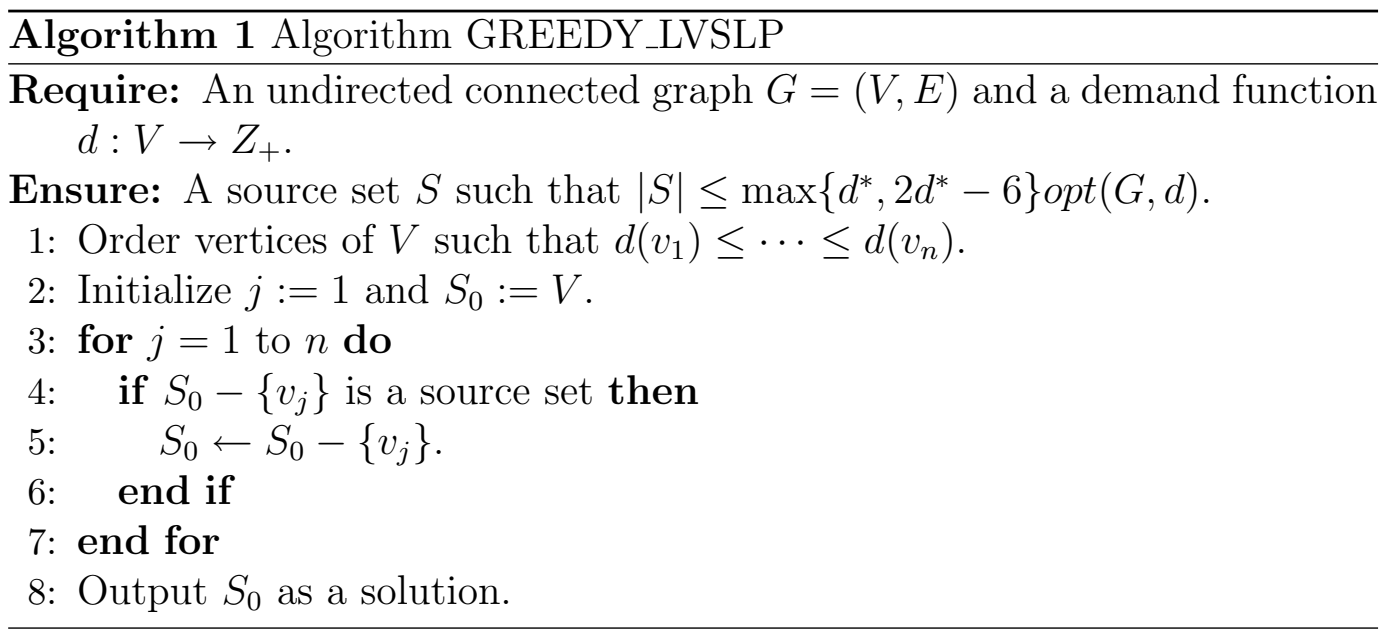

Let $S_{0}=\left\{s_{1}, s_{2}, \ldots, s_{p}\right\}$ be a source set obtained by the algorithm. Here we observe the following property, which will be used for proving the approximation results.

Lemma 10 For each $s \in S_{0}$, there is a deficient set $W$ satisfying the following conditions $(i)-($ iii) :

(i) $W \cap S_{0}=\{s\}$.

(ii) $W$ is minimal with respect to $s$.

(iii) $d(W)=d(s)$.

PROOF. From the construction, when the vertex $s$ is picked in lines $4-6$, $S_{0}^{\prime}-\{s\}$ does not satisfy (1) for the current source set $S_{0}^{\prime}$. Before deleting $s$ from $S_{0}^{\prime}, S_{0}^{\prime}$ is feasible and hence by Lemma 6, every deficient set contains a source in $S_{0}^{\prime}$. On the other hand, $S_{0}^{\prime}-\{s\}$ is infeasible. Again by Lemma 6, there is a deficient set $W$ with $W \cap S_{0}^{\prime}=\{s\}$ such that $W-\{s\}$ is not deficient. Moreover, since all vertices in $W-\{s\}$ have been already deleted, we can observe that $d(s)=\max \{d(v) \mid v \in W\}=d(W)$ holds by the sorting in line 1 , and that $d(s)>\left|N_{G}(W)\right|$. It follows that there is a minimal deficient set $W$ with respect to $s$ satisfying $W \cap S_{0}^{\prime}=\{s\}$ and $d(W)=d(s)$. Moreover, by $S_{0} \subseteq S_{0}^{\prime}$, we have $W \cap S_{0}=\{s\}$.

By this lemma and observations in its proof, we can see that for $s$ and $S_{0}^{\prime}$ defined in the proof, if there is no deficient set $W$ with respect to $s$ such that $W \cap S_{0}^{\prime}=\{s\}$ (i.e., $\left.\hat{\kappa}_{G}\left(S_{0}^{\prime}-\{s\}, s\right) \geq d(s)\right)$, then $S_{0}^{\prime}-\{s\}$ is a source set. Hence, we also have the following lemma.

Lemma 11 In lines $4-6$ of the algorithm GREEDY_LVSLP, a vertex set $S_{0}-$ $\left\{v_{j}\right\}$ is a source set if and only if $\hat{\kappa}_{G}\left(S_{0}-\left\{v_{j}\right\}, v_{j}\right) \geq d\left(v_{j}\right)$.

Let $\mathcal{W}_{0}=\left\{W_{1}, W_{2}, \ldots, W_{p}\right\}$ be a family of deficient sets such that $W_{i}$ satifies 
(i)-(iii) of Lemma 10 for $s_{i} \in S_{0}$. Here we observe that $S_{0}$ is $\max \left\{d^{*}, 2 d^{*}-6\right\}$ approximate.

Lemma $12\left|S_{0}\right| \leq \max \left\{d^{*}, 2 d^{*}-6\right\} \operatorname{opt}(G, d)$.

PROOF. If $d^{*}=1$, then $\left|S_{0}\right|=1$ clearly holds; $S_{0}$ is optimal. Consider the case where $d^{*} \geq 2$. Let $S$ be an arbitrary source set. From the definition of $f\left(V, \mathcal{W}_{0}\right)$, we can observe that $S$ can cover at most $|S| f\left(V, \mathcal{W}_{0}\right)$ sets in $\mathcal{W}_{0}$. On the other hand, Lemma 6 indicates that we have $S \cap W \neq \emptyset$ for every $W \in \mathcal{W}_{0}$. Therefore, $|S| f\left(V, \mathcal{W}_{0}\right) \geq\left|\mathcal{W}_{0}\right|$ must hold. It follows that we have $\operatorname{opt}(G, d) \geq\left|\mathcal{W}_{0}\right| / f\left(V, \mathcal{W}_{0}\right)=\left|S_{0}\right| / f\left(V, \mathcal{W}_{0}\right)$. We will prove this lemma by showing that if $f\left(V, \mathcal{W}_{0}\right) \geq d^{*}+1$, then $f\left(V, \mathcal{W}_{0}\right) \leq 2 d^{*}-5$ and that when $f\left(V, \mathcal{W}_{0}\right)=2 d^{*}-5 \geq d^{*}+1$, we have $\operatorname{opt}(G, d)<2 d^{*}-5$.

Assume that there is a family $\mathcal{W}^{\prime} \subseteq \mathcal{W}_{0}$ of deficient sets with $\left|\mathcal{W}^{\prime}\right|=\ell, \ell \geq$ $d^{*}+1$, and $\bigcap_{W \in \mathcal{W}^{\prime}} W \neq \emptyset$. We first claim that for each $W \in \mathcal{W}^{\prime}$, the number of sets $W_{i} \in \mathcal{W}^{\prime}$ with $s_{i} \in N_{G}(W)$ is at most $d^{*}-3$. From $\left|N_{G}(W)\right| \leq d^{*}-1$, $\ell \geq d^{*}+1$, and Lemma 10(i), there exists a set $W_{j} \in \mathcal{W}^{\prime}$ with $s_{j} \notin N_{G}(W)$ (notice that $\left|N_{G}(W)\right| \leq d^{*}-1$ holds for every $W \in \mathcal{W}_{0}$ since $W$ is deficient and $d^{*}=\max \{d(v) \mid v \in V\}$ ). Again by Lemma 10(i), if this claim would not hold, then such $W_{j}$ would satisfy $\left|W_{j} \cap N_{G}(W)\right| \leq 1$. Then $\left|W_{j} \cap N_{G}(W)\right|=0$ would imply that $W_{j}-W$ and $W_{j} \cap W$ are disconnected, which contradicts Lemma 7 , and $\left|W_{j} \cap N_{G}(W)\right|=1$ would indicate that the vertex $v$ with $W_{j} \cap N_{G}(W)=\{v\}$ satisfies $v=s_{j}$ by Lemmas 8 and 10(ii) (note that $W \cap N_{G}\left(W_{j}\right) \neq \emptyset$ holds by Lemma 7$)$.

Consider the directed graph $H=\left(V_{1}, E_{1}\right)$ such that each vertex $v_{i} \in V_{1}$ corresponds to a set in $W_{i} \in \mathcal{W}^{\prime}$, and that a directed edge $\left(v_{i}, v_{j}\right)$ belongs to $E_{1}$ if and only if $s_{j} \in N_{G}\left(W_{i}\right)$. From the above claim, the outdegree of each vertex in $V_{1}$ is at most $d^{*}-3$. On the other hand, $\left|E_{1}\right| \geq \ell(\ell-1) / 2$ holds, since Lemmas 9 and 10 imply that for every two sets $W_{i}, W_{j} \in \mathcal{W}^{\prime}$, we have $s_{i} \in N_{G}\left(W_{j}\right)$ or $s_{j} \in N_{G}\left(W_{i}\right)$. It follows that $\left(d^{*}-3\right) \ell \geq\left|E_{1}\right| \geq \ell(\ell-1) / 2$; $\ell \leq 2 d^{*}-5$.

Finally, we consider a special case of $\ell=2 d^{*}-5$. Then, by the above inequality, the outdegree of each vertex in $V_{1}$ is exactly $d^{*}-3$. Now notice that every $W_{i} \in$ $\mathcal{W}^{\prime}$ satisfies $\left|N_{G}\left(W_{i}\right)\right| \leq d^{*}-1$ and $\left|N_{G}\left(W_{i}\right) \cap W_{j}\right| \geq 2$ for each $W_{j} \in \mathcal{W}^{\prime}-\left\{W_{i}\right\}$ with $s_{j} \notin N_{G}\left(W_{i}\right)$ as observed above. It follows that we have $\left|N_{G}\left(W_{i}\right)\right|=$ $d^{*}-1$ and $N_{G}\left(W_{i}\right) \subseteq \bigcup_{W \in \mathcal{W}^{\prime}} W$ for each $W_{i} \in \mathcal{W}^{\prime} ; N_{G}\left(\bigcup_{W \in \mathcal{W}^{\prime}} W\right)=\emptyset$, $V=\bigcup_{W \in \mathcal{W}^{\prime}} W, \mathcal{W}_{0}=\mathcal{W}^{\prime}$, and each $s_{i} \in S_{0}$ satisfies $d\left(s_{i}\right)=d^{*}$ (note that $G$ is connected and that by $V=\bigcup_{W \in \mathcal{W}^{\prime}} W$ and Lemma 10(i), any set in $\mathcal{W}_{0}-\mathcal{W}^{\prime}$ cannot exist). Observe that $\operatorname{opt}(G, d) \geq 2$ since if $\{v\}$ would be an optimal solution for some $v \in V$, then $V-\{v\}$ would be a deficient set with respect to some $s \in S_{0}-\{v\}$ and hence $\{v\}$ would be infeasible (note that 
$\left|N_{G}(V-\{v\})\right|=1<d^{*}$ and $\left.\left|S_{0}\right| \geq d^{*}+1>1\right)$. It follows that if $\ell=2 d^{*}-5$, $\left|S_{0}\right|=\ell \leq\left(2 d^{*}-5\right) \operatorname{opt}(G, d) / 2$.

Finally, we show that the algorithm GREEDY_LVSLP can be implemented to run in $O\left(\min \left\{d^{*}, \sqrt{n}\right\} d^{*} n^{2}\right)$ time. From Lemma 11 , we can observe that the procedure in lines $4-6$ can be done in $O\left(\min \left\{d^{*}, \sqrt{n}\right\} m\right)$ time by using the network flow computation [4]. Since the procedure in lines $4-6$ is executed at most $n$ times, it follows that the total complexity is $O\left(\min \left\{d^{*}, \sqrt{n}\right\} m n\right)$.

Moreover, it was shown in [13] that for any graph $H$ and any integer $k$, a sparse subgraph $H_{k}$ of $H$ with $O\left(d^{*} n\right)$ edges satisfying the following (i) and (ii) can be obtained in $O(|E(H)|)$ time. (i) The local vertex-connectivity less than $k$ in $H$ is preserved also in $H_{k}$. (ii) The local vertex-connectivity at least $k$ in $H$ is at least $k$ also in $H_{k}$. Notice that since $d^{*}$ is the maximum demand, what we need to concern is the connectivity less than $d^{*}$. Hence, by computing such a sparse subgraph $G_{d^{*}}$ of $G$ with $O\left(d^{*} n\right)$ edges and applying the algorithm GREEDY_LVSLP to this $G_{d^{*}}$, we can reduce the above complexity to $O(m+$ $\left.\min \left\{d^{*}, \sqrt{n}\right\} d^{*} n^{2}\right)$.

Summarizing the arguments given so far, Theorem 4 is now established.

\section{The case of $d^{*} \leq 4$}

In this section, we consider 4LVSLP. Let $S_{0}$ and $\mathcal{W}_{0}$ be a set of vertices obtained by algorithm GREEDY_LVSLP and the family of deficient sets corresponding to $S_{0}$, respectively, as defined in the previous section. Here we show that $S_{0}$ is 3-approximate and that this analysis is tight for the algorithm. We also show that 4LVSLP is APX-hard.

Assume that $d^{*}=4$, since the case of $d^{*} \leq 3$ is solvable in polynomial time, as shown in [7]. Also assume that $\left|S_{0}\right| \geq 4$, since $\left|S_{0}\right| \leq 3$ implies that $S_{0}$ is 3 -approximate. If the frequency of each vertex with respect to $\mathcal{W}_{0}$ is at most three, then $S_{0}$ is 3-approximate as observed in the proof of Lemma 12. However, there exists an instance which has a vertex with frequency four. We first start with characterizing such cases through the following preparatory lemmas.

Lemma 13 Let $W_{i}$ and $W_{j}$ denote deficient sets in $\mathcal{W}_{0}$ with $W_{i} \cap W_{j} \neq \emptyset$.

(i) $\left|N_{G}\left(W_{i} \cup W_{j}\right)\right| \geq 1$.

(ii) $W_{i} \cap N_{G}\left(W_{j}\right) \neq \emptyset \neq W_{j} \cap N_{G}\left(W_{i}\right)$ holds; $\left|N_{G}\left(W_{i} \cap W_{j}\right)\right| \geq 2$.

(iii) If $\left|N_{G}\left(W_{i} \cap W_{j}\right)\right|=2$, then no set $W \in \mathcal{W}_{0}-\left\{W_{i}, W_{j}\right\}$ satisfies $W \cap$ $W_{i} \cap W_{j} \neq \emptyset$. 
(iv) If $\left|N_{G}\left(W_{i} \cup W_{j}\right)\right|=1$, then at most one set $W \in \mathcal{W}_{0}-\left\{W_{i}, W_{j}\right\}$ satisfies $W \cap W_{i} \cap W_{j} \neq \emptyset$.

(v) If $\left|N_{G}\left(W_{i} \cup W_{j}\right)\right|=2$, then for every $W \in \mathcal{W}_{0}-\left\{W_{i}, W_{j}\right\}$ with $W_{i} \cap W_{j} \cap$ $W \neq \emptyset$, we have $N_{G}\left(W_{i} \cup W_{j}\right) \cap W \cap S_{0} \neq \emptyset$.

PROOF. (i) Lemma 10(i) implies that $S_{0}-\left\{s_{i}, s_{j}\right\} \subseteq V-\left(W_{i} \cup W_{j}\right)$ (note that $S_{0}-\left\{s_{i}, s_{j}\right\} \neq \emptyset$ by $\left.\left|S_{0}\right| \geq 4\right)$. Hence, $N_{G}\left(W_{i} \cup W_{j}\right)=\emptyset$ would contradict the connectedness of $G$.

(ii) This follows from Lemma 7.

(iii) By (ii), we have $\left|W_{i} \cap N_{G}\left(W_{j}\right)\right|=\left|W_{j} \cap N_{G}\left(W_{i}\right)\right|=1$. It follows from Lemma 8 that $W_{i} \cap N_{G}\left(W_{j}\right)=\left\{s_{i}\right\}$ and $W_{j} \cap N_{G}\left(W_{i}\right)=\left\{s_{j}\right\} ; N_{G}\left(W_{i} \cap W_{j}\right)=$ $\left\{s_{i}, s_{j}\right\}$. Lemma 10(i) indicates that any set $W \in \mathcal{W}_{0}-\left\{W_{i}, W_{j}\right\}$ satisfies $W \cap N_{G}\left(W_{i} \cap W_{j}\right)=\emptyset$ and $W-\left(W_{i} \cup W_{j}\right) \neq \emptyset$. Hence, we can observe that no set $W \in \mathcal{W}_{0}-\left\{W_{i}, W_{j}\right\}$ satisfies $W \cap W_{i} \cap W_{j} \neq \emptyset$, since if such a set $W$ would exist, then $G[W]$ would be disconnected, contradicting Lemma 7.

(iv) Let $\{v\}=N_{G}\left(W_{i} \cup W_{j}\right)$. Assume that there is a set $W_{\ell} \in \mathcal{W}_{0}-\left\{W_{i}, W_{j}\right\}$ with $W_{i} \cap W_{j} \cap W_{\ell} \neq \emptyset$. Then by applying Lemma 8 as $X=W_{i} \cup W_{j}$ and $W=W_{\ell}$, we have $v=s_{\ell}$. Hence, from Lemmas 7 and 10, we can observe that for any set $W \in \mathcal{W}_{0}-\left\{W_{i}, W_{j}, W_{\ell}\right\}$, we have $W \cap W_{i} \cap W_{j}=\emptyset$.

(v) Let $W_{\ell} \in \mathcal{W}_{0}-\left\{W_{i}, W_{j}\right\}$ be a set with $W_{i} \cap W_{j} \cap W_{\ell} \neq \emptyset$. If $\mid N_{G}\left(W_{i} \cup\right.$ $\left.W_{j}\right) \cap W_{\ell} \mid=1$, then $N_{G}\left(W_{i} \cup W_{j}\right) \cap W_{\ell}=\left\{s_{\ell}\right\}$ holds by Lemma 8. Consider the case where $\left|N_{G}\left(W_{i} \cup W_{j}\right) \cap W_{\ell}\right|=2 ; N_{G}\left(W_{i} \cup W_{j}\right) \subseteq W_{\ell}$. Assume by contradiction that $s_{\ell} \in W_{\ell}-\left(W_{i} \cup W_{j} \cup N_{G}\left(W_{i} \cup W_{j}\right)\right)$ holds. Lemma 9 indicates that $\left\{s_{i}, s_{j}\right\} \subseteq N_{G}\left(W_{\ell}\right)$. From the connectedness of $G,\left|S_{0}\right| \geq 4$, and $N_{G}\left(W_{i} \cup W_{j}\right) \subseteq W_{\ell}$, we can observe that $N_{G}\left(W_{\ell}\right)-\left(W_{i} \cup W_{j}\right) \neq \emptyset$. It follows from $\left|N_{G}\left(W_{\ell}\right)\right| \leq d^{*}-1=3$ that $\left|N_{G}\left(W_{\ell}\right)\right|=3$ and $d\left(s_{\ell}\right)=4$; $N_{G}\left(W_{\ell}\right) \cap\left(W_{i} \cup W_{j}\right)=\left\{s_{i}, s_{j}\right\}$ and $\left|N_{G}\left(W_{\ell}\right)-\left(W_{i} \cup W_{j}\right)\right|=1$. Moreover, $N_{G}\left(W_{\ell}-\left(W_{i} \cup W_{j} \cup N_{G}\left(W_{i} \cup W_{j}\right)\right)\right) \subseteq N_{G}\left(W_{i} \cup W_{j}\right) \cup\left(N_{G}\left(W_{\ell}\right)-\left(W_{i} \cup W_{j}\right)\right)$ and hence $\left|N_{G}\left(W_{\ell}-\left(W_{i} \cup W_{j} \cup N_{G}\left(W_{i} \cup W_{j}\right)\right)\right)\right| \leq\left|N_{G}\left(W_{i} \cup W_{j}\right)\right|+\mid N_{G}\left(W_{\ell}\right)-$ $\left(W_{i} \cup W_{j}\right) \mid \leq 3$, contradicting the minimality of $W_{\ell}$.

Lemma $14 f\left(V, \mathcal{W}_{0}\right) \leq 4$ holds. In particular, for a vertex $v \in V$ whose frequency is four, the four distinct sets $W_{i} \in \mathcal{W}_{0}, i=1,2,3,4$ with $v \in W_{i}$ satisfy the following (4):

For some two sets $W_{1}, W_{2}, d\left(s_{1}\right)=4$ and $d\left(s_{2}\right) \geq 3$ hold and any set in $\mathcal{W}_{0}-\left\{W_{1}, W_{2}, W_{3}, W_{4}\right\}$ is disjoint with $W_{1} \cup W_{2}$.

PROOF. Let $W_{1}$ and $W_{2}$ denote deficient sets in $\mathcal{W}_{0}$ with $W_{1} \cap W_{2} \neq \emptyset$. 
We observe how many sets in $\mathcal{W}_{0}-\left\{W_{1}, W_{2}\right\}$ can intersect with $W_{1} \cap W_{2}$. From Lemma 13(i)(ii), we have $\left|N_{G}\left(W_{1} \cup W_{2}\right)\right| \geq 1$ and $\left|N_{G}\left(W_{1} \cap W_{2}\right)\right| \geq 2$. Moreover, Lemma 13(iii) says that if $\left|N_{G}\left(W_{1} \cap W_{2}\right)\right|=2$, then every set $W \in \mathcal{W}_{0}-\left\{W_{1}, W_{2}\right\}$ is disjoint with $W_{1} \cap W_{2}$.

Consider the case where $\left|N_{G}\left(W_{1} \cap W_{2}\right)\right| \geq 3$. By (2) and $\left|N_{G}(W)\right| \leq d^{*}-$ $1 \leq 3$ for each $W \in \mathcal{W}_{0}$, we have $\left|N_{G}\left(W_{1} \cup W_{2}\right)\right| \leq 3$. In particular, if $\left|N_{G}\left(W_{1} \cup W_{2}\right)\right|=3$ (resp. $\left|N_{G}\left(W_{1} \cup W_{2}\right)\right|=2$ ), then we have $\left|N_{G}\left(W_{1}\right)\right|=$ $\left|N_{G}\left(W_{2}\right)\right|=\left|N_{G}\left(W_{1} \cap W_{2}\right)\right|=3$ (resp. $\left|N_{G}\left(W_{1}\right)\right|=3$ and $\left|N_{G}\left(W_{2}\right)\right| \geq 2$ without loss of generality). There are the following three possible cases (I) $\left|N_{G}\left(W_{1} \cup W_{2}\right)\right|=1$, (II) $\left|N_{G}\left(W_{1} \cup W_{2}\right)\right|=2,\left|N_{G}\left(W_{1}\right)\right|=3$, and $\left|N_{G}\left(W_{2}\right)\right| \geq 2$, and (III) $\left|N_{G}\left(W_{1} \cup W_{2}\right)\right|=3$ and $\left|N_{G}\left(W_{1}\right)\right|=\left|N_{G}\left(W_{2}\right)\right|=\left|N_{G}\left(W_{1} \cap W_{2}\right)\right|=3$.

(I) Lemma 13(iv) implies that the frequency of each vertex in $W_{1} \cap W_{2}$ is at most three.

(II) Assume that there are two distinct sets $W_{3}, W_{4} \in \mathcal{W}_{0}-\left\{W_{1}, W_{2}\right\}$ such that $W_{1} \cap W_{2} \cap W_{3} \cap W_{4} \neq \emptyset$. Lemma 13(v) implies that $N_{G}\left(W_{1} \cup W_{2}\right)=$ $\left\{s_{3}, s_{4}\right\}$. Hence, any other set $W \in \mathcal{W}_{0}$ cannot intersect with $W_{1} \cup W_{2}$ by $W \cap\left\{s_{3}, s_{4}\right\}=\emptyset$ and the connectedness of $G[W]$. Therefore, we can observe that the frequency of each vertex in $W_{1} \cap W_{2}$ is at most four and that if $W_{1} \cap W_{2} \cap W_{3} \cap W_{4} \neq \emptyset$ holds, then (4) holds.

(III) Assume that there is a set $W_{3} \in \mathcal{W}_{0}-\left\{W_{1}, W_{2}\right\}$ with $W_{1} \cap W_{2} \cap W_{3} \neq$ $\emptyset$. We also assume that $\left|N_{G}\left(W_{3} \cup W_{1}\right)\right|=\left|N_{G}\left(W_{2} \cup W_{3}\right)\right|=3$ and hence $\left|N_{G}\left(W_{3}\right)\right|=3$, since otherwise we can apply the above arguments. Note that $d\left(s_{1}\right)=d\left(s_{2}\right)=d\left(s_{3}\right)=4$. Then we have the following claim, which proves this lemma.

Claim 15 Every set in $\mathcal{W}_{0}-\left\{W_{1}, W_{2}, W_{3}\right\}$ is disjoint with $W_{1} \cap W_{2} \cap W_{3}$.

PROOF. We have $\left|N_{G}\left(W_{3}\right) \cap\left(W_{1} \cup W_{2}\right)\right| \geq 2$, since otherwise if $\mid N_{G}\left(W_{3}\right) \cap$ $\left(W_{1} \cup W_{2}\right) \mid=1$ would hold, then Lemma 8 would indicate that $N_{G}\left(W_{3}\right) \cap$ $\left(W_{1} \cup W_{2}\right)=\left\{s_{1}\right\}=\left\{s_{2}\right\}$, a contradiction.

Now by $\left|N_{G}\left(W_{1} \cap W_{2}\right)\right|=3$, there are the following two possible cases: (III-1) $\left|N_{G}\left(W_{1}\right) \cap W_{2}\right|=\left|N_{G}\left(W_{2}\right) \cap W_{1}\right|=\left|N_{G}\left(W_{1} \cap W_{2}\right)-\left(W_{1} \cup W_{2}\right)\right|=1$, (III-2) $\left|N_{G}\left(W_{1}\right) \cap W_{2}\right|=2$ and $\left|N_{G}\left(W_{2}\right) \cap W_{1}\right|=1$ without loss of generality. In both cases, we have $N_{G}\left(W_{2}\right) \cap W_{1}=\left\{s_{1}\right\}$.

(III-1) By Lemma 8, we have $N_{G}\left(W_{1}\right) \cap W_{2}=\left\{s_{2}\right\}$. Let $\left\{v_{12}\right\}=N_{G}\left(W_{1} \cap W_{2}\right)-$ $\left(W_{1} \cup W_{2}\right)$. By the connectedness of $G\left[W_{3}\right]$ and $\left\{s_{1}, s_{2}\right\} \cap W_{3}=\emptyset$, we have $v_{12} \in$ $W_{3}$. If $v_{12}=s_{3}$, then $N_{G}\left(W_{1} \cap W_{2}\right)=\left\{s_{1}, s_{2}, s_{3}\right\}$ holds and hence every set in $\mathcal{W}_{0}-\left\{W_{1}, W_{2}, W_{3}\right\}$ is disjoint with $W_{1} \cap W_{2}$. Consider the case where $v_{12} \neq s_{3}$. Note that from the connectedness of $G\left[W_{1}\right], N_{G}\left(W_{3}\right) \cap\left(\left(W_{1} \cap W_{2}\right) \cup\left\{s_{1}\right\}\right) \neq \emptyset$ 
holds. Then $\left(W_{1} \cap W_{2}\right) \cup\left\{s_{1}\right\}$ has a neighbour in $W_{3}$ other than $v_{12}$, since if $N_{G}\left(\left(W_{1} \cap W_{2}\right) \cup\left\{s_{1}\right\}\right) \cap W_{3}=\left\{v_{12}\right\}$ would hold, then by applying Lemma 8 as $X=\left(W_{1} \cap W_{2}\right) \cup\left\{s_{1}\right\}$ and $W=W_{3}, v_{12}=s_{3}$ would hold. It follows that $s_{1} \in N_{G}\left(W_{3}\right)$. Similarly, $s_{2} \in N_{G}\left(W_{3}\right)$ holds. On the other hand, we have $N_{G}\left(W_{2}\right)-\left\{s_{1}\right\} \subseteq W_{3}$, since otherwise $\left|N_{G}\left(W_{2}\right) \cap W_{3}\right|=\left|\left\{v_{12}\right\}\right|=1$ would hold and Lemma 8 would imply that $v_{12}=s_{3}$. It follows that $N_{G}\left(W_{2}\right) \subseteq$ $W_{3} \cup N_{G}\left(W_{3}\right)$ and $\left|N_{G}\left(W_{3}\right)-W_{2}\right| \leq 2$. Therefore, we have $\left|N_{G}\left(W_{2} \cup W_{3}\right)\right| \leq 2$, contradicting the assumption.

(III-2) Let $\left\{v_{1}, v_{2}\right\}=N_{G}\left(W_{1}\right) \cap W_{2},\left\{v_{3}\right\}=N_{G}\left(W_{1}\right)-\left\{v_{1}, v_{2}\right\}$, and $\left\{v_{4}, v_{5}\right\}=$ $N_{G}\left(W_{2}\right)-\left\{s_{1}\right\}$. By the connectedness of $G\left[W_{3}\right]$, we have $\left\{v_{1}, v_{2}\right\} \cap W_{3} \neq \emptyset$ (say, $v_{1} \in W_{3}$ ) and $\left\{v_{4}, v_{5}\right\} \cap W_{3} \neq \emptyset$ (say, $v_{4} \in W_{3}$ ). Then we claim that $\left(W_{1} \cap W_{2}\right) \cup\left\{s_{1}\right\}$ has a neighbour in $W_{3}$ other than $v_{1}$, since if $\mid N_{G}\left(\left(W_{1} \cap\right.\right.$ $\left.\left.W_{2}\right) \cup\left\{s_{1}\right\}\right) \cap W_{3} \mid=1$, then by applying Lemma 8 as $X=\left(W_{1} \cap W_{2}\right) \cup\left\{s_{1}\right\}$ and $W=W_{3}, s_{3}=v_{1} \in W_{2}$ would hold, contradicting Lemma 10(i) (note that $N_{G}\left(W_{3}\right) \cap\left(\left(W_{1} \cap W_{2}\right) \cup\left\{s_{1}\right\}\right) \neq \emptyset$ by the connectedness of $\left.G\left[W_{1}\right]\right)$.

Here we claim that $s_{1} \in N_{G}\left(W_{3}\right)$. If $v_{2} \notin W_{3}$, then $\left(N_{G}\left(\left(W_{1} \cap W_{2}\right) \cup\left\{s_{1}\right\}\right) \cap\right.$ $\left.W_{3}\right)-\left\{v_{1}\right\}$ is included in $\left(\left(W_{1}-W_{2}\right)-\left\{s_{1}\right\}\right) \cup\left\{v_{3}\right\} ; s_{1} \in N_{G}\left(W_{3}\right)$. If $v_{2} \in W_{3}$, then $\left|N_{G}\left(W_{3}\right) \cap W_{1}\right| \geq 2$ cannot hold, since otherwise $\left|N_{G}\left(W_{1} \cup W_{3}\right)\right| \leq 2$ would hold. It follows from Lemma 8 that $v_{2} \in W_{3}$ indicates $N_{G}\left(W_{3}\right) \cap W_{1}=\left\{s_{1}\right\}$.

We next claim that $N_{G}\left(W_{3}\right)-\left(W_{2} \cup\left\{v_{5}, s_{1}\right\}\right) \neq \emptyset$ and $N_{G}\left(W_{3}\right) \cap\left(W_{2} \cup\left\{v_{5}\right\}\right)=$ $\left\{s_{2}\right\}$. If $N_{G}\left(W_{3}\right) \subseteq W_{2} \cup\left\{v_{5}, s_{1}\right\}$, then it follows that $N_{G}\left(W_{3}\right) \subseteq W_{2} \cup N_{G}\left(W_{2}\right)$ and $\left|N_{G}\left(W_{2} \cup W_{3}\right)\right| \leq 2$, a contradiction. Note that $\left|N_{G}\left(W_{3}\right)-\left\{s_{1}\right\}\right| \leq 2$ and $W_{2} \cap N_{G}\left(W_{3}\right) \neq \emptyset$. Hence, we have $\left|W_{2} \cap N_{G}\left(W_{3}\right)\right|=1, v_{5} \notin N_{G}\left(W_{3}\right)$, and $N_{G}\left(W_{3}\right)-\left(W_{2} \cup\left\{v_{5}, s_{1}\right\}\right) \neq \emptyset$. Moreover, by applying Lemma 8 as $X=W_{3}$ and $W=W_{2}$, we have $N_{G}\left(W_{3}\right) \cap W_{2}=\left\{s_{2}\right\}$.

On the other hand, $v_{5} \notin W_{3}$, since otherwise we would have $N_{G}\left(W_{2}\right) \subseteq W_{3} \cup$ $N_{G}\left(W_{3}\right)$ and $\left|N_{G}\left(W_{2} \cup W_{3}\right)\right| \leq 2$, a contradiction. By Lemma 8 and $W_{3} \cap$ $N_{G}\left(W_{2}\right)=\left\{v_{4}\right\}$, we have $v_{4}=s_{3}$. It follows that $N_{G}\left(W_{2} \cap W_{3}\right)=\left\{s_{1}, s_{2}, s_{3}\right\}$, and every set $W \in \mathcal{W}_{0}-\left\{W_{1}, W_{2}, W_{3}\right\}$ is disjoint with $W_{1} \cap W_{2} \cap W_{3}$.

Lemma 16 Let $W_{i}, W_{j}$ be two minimal deficient sets with respect to $v_{i}$ and $v_{j}$, respectively, such that $W_{i} \cap W_{j} \neq \emptyset,\left|N_{G}\left(W_{i} \cup W_{j}\right)\right| \leq 2, d\left(v_{i}\right)=4$, and $\left\{v_{i}, v_{j}\right\} \cap\left(W_{i} \cap W_{j}\right)=\emptyset$. Then for any feasible solution $S$ to $4 L V S L P$, we have $\left|S \cap\left(W_{i} \cup W_{j}\right)\right| \geq 2$.

PROOF. By Lemma 6, $S \cap\left(W_{i} \cup W_{j}\right) \neq \emptyset$ holds; let $s \in S \cap\left(W_{i} \cup W_{j}\right)$. Now we have $\left|N_{G}\left(W_{i} \cup W_{j}-\{s\}\right)\right| \leq\left|N_{G}\left(W_{i} \cup W_{j}\right)\right|+1 \leq 3<d\left(v_{i}\right)$. Hence, if $s \neq v_{i}$, then again by Lemma 6 , we have $S \cap\left(W_{i} \cup W_{j}-\{s\}\right) \neq \emptyset$ and $\left|S \cap\left(W_{i} \cup W_{j}\right)\right| \geq 2$. If $s=v_{i}$, then $s=v_{i} \notin W_{j}$ holds and hence by Lemma 6 we have $(S-s) \cap W_{j} \neq \emptyset$. Also in this case, $\left|S \cap\left(W_{i} \cup W_{j}\right)\right| \geq 2$. 
Lemma $17 S_{0}$ is 3-approximate.

PROOF. Let $S^{*}$ denote an optimal solution. Since $S^{*}$ is feasible, we have $W \cap S^{*} \neq \emptyset$ for every $W \in \mathcal{W}_{0}$. Consider a mapping $g: \mathcal{W}_{0} \rightarrow S^{*}$ such that for each set $W \in \mathcal{W}_{0}, g(W)=s^{*}$ holds for some source $s^{*} \in S^{*}$ with $s^{*} \in W$. If $\left|\left\{W \in \mathcal{W}_{0} \mid g(W)=s^{*}\right\}\right| \leq 3$ holds for each source $s^{*} \in S^{*}$, then we have $\left|\mathcal{W}_{0}\right| \leq 3\left|S^{*}\right|$, from which $\left|S_{0}\right|=\left|\mathcal{W}_{0}\right| \leq 3\left|S^{*}\right|$. We claim that there is such a mapping.

Assume that for a mapping $g$, there is a source $s_{1}^{*} \in S^{*}$ which at least four sets in $\mathcal{W}_{0}$ is mapped to. By Lemma $14, f\left(V, \mathcal{W}_{0}\right) \leq 4$ holds, and hence the number of sets in $\mathcal{W}_{0}$ mapped to $s_{1}^{*}$ is exactly four. Moreover, the four sets $W_{1}, W_{2}, W_{3}, W_{4}$ in $\mathcal{W}_{0}$ with $g\left(W_{i}\right)=s_{1}^{*}, i=1,2,3,4$ satisfy (4); $\left|N_{G}\left(W_{1} \cup W_{2}\right)\right|=2, d\left(s_{1}\right)=4$, and $W \cap\left(W_{1} \cup W_{2}\right)=\emptyset$ for each $W \in \mathcal{W}_{0}-\left\{W_{1}, W_{2}, W_{3}, W_{4}\right\}$ (notice that in this case, $\left|N_{G}\left(W_{1} \cup W_{2}\right)\right|=2$ holds by the proof of Lemma 14).

Now Lemma 16 implies that $W_{1} \cup W_{2}$ includes a source $s_{2}^{*} \in S^{*}-\left\{s_{1}^{*}\right\}$. Notice that no set in $\mathcal{W}_{0}$ is mapped to $s_{2}^{*}$ in $g$ because every set $W \in$ $\mathcal{W}_{0}-\left\{W_{1}, W_{2}, W_{3}, W_{4}\right\}$ satisfies $s_{2}^{*} \notin W$ and each of $W_{i}, i=1,2,3,4$ has been mapped to $s_{1}^{*}$. So, we can decrease the number of sets in $\mathcal{W}_{0}$ mapped to $s_{1}^{*}$ by one, by remapping one of two sets $W_{1}$ and $W_{2}$ including $s_{2}^{*}$ to $s_{2}^{*}$. Consequently, by repeating this arguments, we can obtain a mapping with the required property.

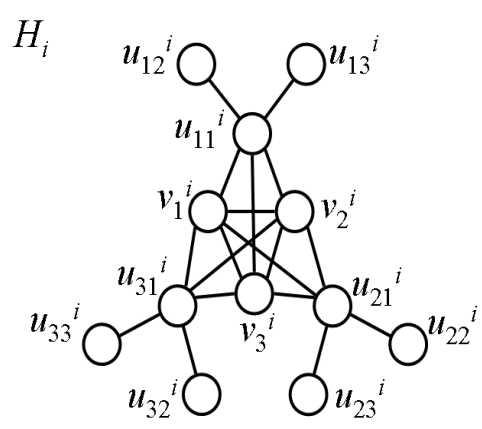

(a)

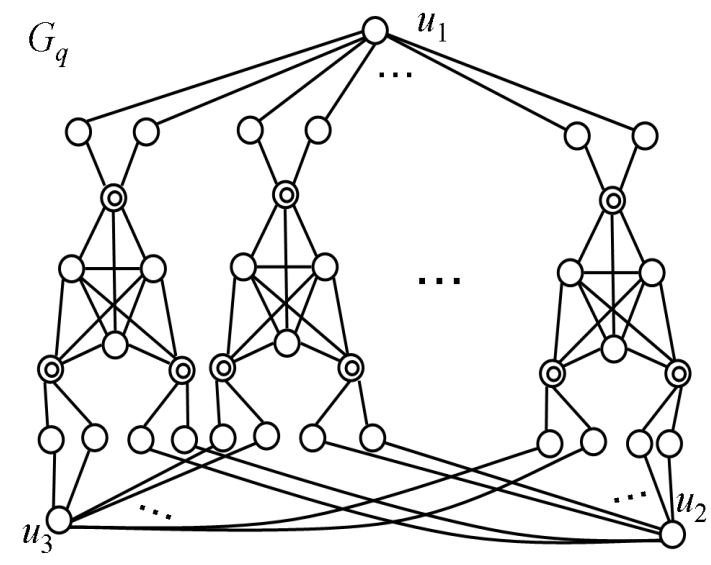

(b)

Fig. 1. Illustration of a tight example $G$ for the algorithm GREEDY_LVSLP in 4LVSLP. (a) shows the graph $H_{i}$ which is a subgraph of $G$ in (b). In $G$, each vertex $v$ with $d(v)=4$ is drawn as double circles. 
We now give a tight example for the algorithm GREEDY_LVSLP. Let $H_{i}=$ $\left(V_{i}, E_{i}\right)$ be the graph where $V_{i}=\bigcup_{j=1}^{3}\left\{v_{j}^{i}, u_{j 1}^{i}, u_{j 2}^{i}, u_{j 3}^{i}\right\}$ and $E_{i}=\left(\bigcup_{j, \ell}\left(v_{j}^{i}, v_{\ell}^{i}\right)\right)$ $\cup\left(\bigcup_{j=1}^{3}\left\{\left(u_{j 1}^{i}, u_{j 2}^{i}\right),\left(u_{j 1}^{i}, u_{j 3}^{i}\right),\left(u_{j 1}^{i}, v_{1}^{i}\right),\left(u_{j 1}^{i}, v_{2}^{i}\right),\left(u_{j 1}^{i}, v_{3}^{i}\right)\right\}\right)$ (see Fig. 1(a)). Let $G_{q}=(V, E)$ be the graph where $V=\left\{u_{1}, u_{2}, u_{3}\right\} \cup\left(\bigcup_{i=1}^{q} V_{i}\right), q \geq 4$ and $E=$ $\bigcup_{i=1}^{q}\left(E_{i} \cup\left(\bigcup_{j=1}^{3}\left\{\left(u_{j}, u_{j 2}^{i}\right),\left(u_{j}, u_{j 3}^{i}\right)\right\}\right)\right), d\left(u_{j 1}^{i}\right)=4$ for each $i \in\{1,2, \ldots, q\}$ and $j \in\{1,2,3\}$, and $d(v)=0$ for all other vertices (see Fig. 1(b)). For $G_{q}$ and $d$, the algorithm GREEDY_LVSLP returns a source set $S_{0}=\bigcup_{i=1}^{q}\left\{u_{11}^{i}, u_{21}^{i}, u_{31}^{i}\right\}$ and $\mathcal{W}_{0}=\bigcup_{i=1}^{q}\left\{\left\{u_{11}^{i}, u_{12}^{i}, u_{13}^{i}, v_{1}^{i}, v_{2}^{i}, v_{3}^{i}\right\},\left\{u_{21}^{i}, u_{22}^{i}, u_{23}^{i}, v_{1}^{i}, v_{2}^{i}, v_{3}^{i}\right\},\left\{u_{31}^{i}, u_{32}^{i}, u_{33}^{i}\right.\right.$, $\left.\left.v_{1}^{i}, v_{2}^{i}, v_{3}^{i}\right\}\right\}$. On the other hand, $\left\{v_{1}^{1}, v_{1}^{2}, \ldots, v_{1}^{q}\right\}$ is an optimal solution. This example shows that our analysis of the algorithm is tight. Here we remark that in a similar way, we can construct an instance in which GREEDY_LVSLP returns a solution $S$ with $|S|=\left(d^{*}-1\right) \operatorname{opt}(G, d)$ for a general $d$. Namely, we have the following lemma.

Lemma 18 For $d^{*} L V S L P$, there exists a graph for which the algorithm GREEDY_LVSLP provides no better than a $\left(d^{*}-1\right)$-approximate solution. In particular, for $4 L V S L P$, such a graph is a tight example. A graph in Fig. 1 (b) is one of such examples.

Finally, we show that the problem is APX-hard. In [7], it was shown that 4LVSLP is NP-hard by a reduction from the minimum vertex cover problem restricted to 3-regular graphs:

\section{Vertex-cover problem in a 3-regular graph (VC3R)}

INSTANCE: $(G=(V, E), k)$ : A 3-regular graph $G=(V, E)$ and an integer $k$.

QUESTION: Is there a vertex cover $X$ with $|X| \leq k$ in $G$ ?

where a set $V^{\prime} \subseteq V$ of vertices is called a vertex cover if every edge $e=$ $(u, v) \in E$ satisfies $\{u, v\} \cap V^{\prime} \neq \emptyset$, and a graph is called $k$-regular if the degree of every vertex is exactly $k$. As shown in [1], the minimum vertex cover problem is APX-hard, even restricted to 3-regular graphs. We can prove the APX-hardness of 4LVSLP by using the same reduction as [7].

Lemma $194 L V S L P$ is $A P X$-hard.

PROOF. We start with reviewing a reduction from the minimum vertex cover problem in a 3-regular graph to 4LVSLP, which was shown in [7].

Take an instance $I_{V C 3 R}=\left(G_{1}=\left(V_{1}, E_{1}\right), k\right)$ of VC3R, where $n_{1}=\left|V_{1}\right|$ and $m_{1}=\left|E_{1}\right|$. Let $G_{2}=\left(V_{2}, E_{2}\right)$ be the graph obtained from $G_{1}$ by replacing each edge $e=\left(v_{i}, v_{j}\right) \in E_{1}$ with three edges $\left(v_{i}, v_{i, j}\right),\left(v_{i, j}, v_{j, i}\right)$, and $\left(v_{j, i}, v_{j}\right)$ introducing two new vertices $v_{i, j}$ and $v_{j, i} ; V_{2}=V_{1} \cup V_{2, E}$ and 

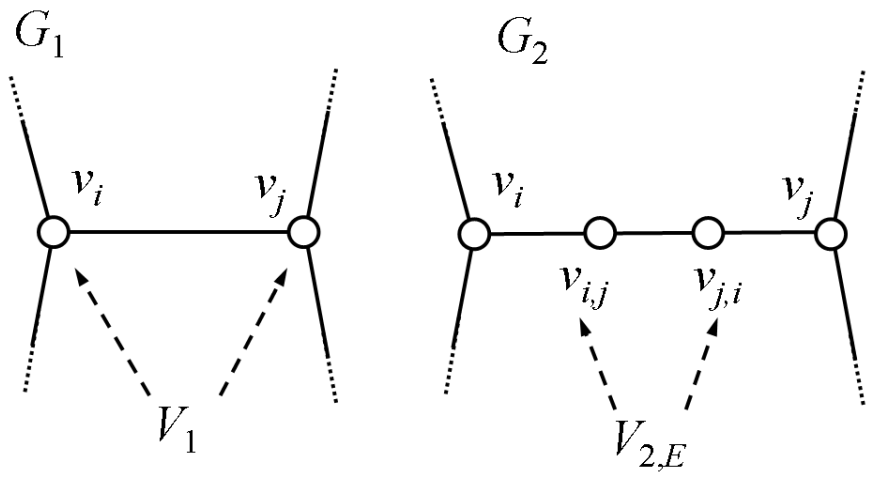

Fig. 2. Illustration of an edge $\left(v_{i}, v_{j}\right)$ in $G_{1}$ and the corresponding edges in $G_{2}$ in the proof of Lemma 19.

$E_{2}=\cup_{\left(v_{i}, v_{j}\right) \in E_{1}}\left\{\left(v_{i}, v_{i, j}\right),\left(v_{i, j}, v_{j, i}\right),\left(v_{j, i}, v_{j}\right)\right\}$, where $V_{2, E}=\bigcup_{\left(v_{i}, v_{j}\right) \in E_{1}}\left\{v_{i, j}, v_{j, i}\right\}$ (see Fig. 2). From $G_{2}$, we construct an instance $I_{L V S L P}=\left(G_{3}=\left(V_{3}, E_{3}\right), d\right)$ of 4LVSLP as follows.

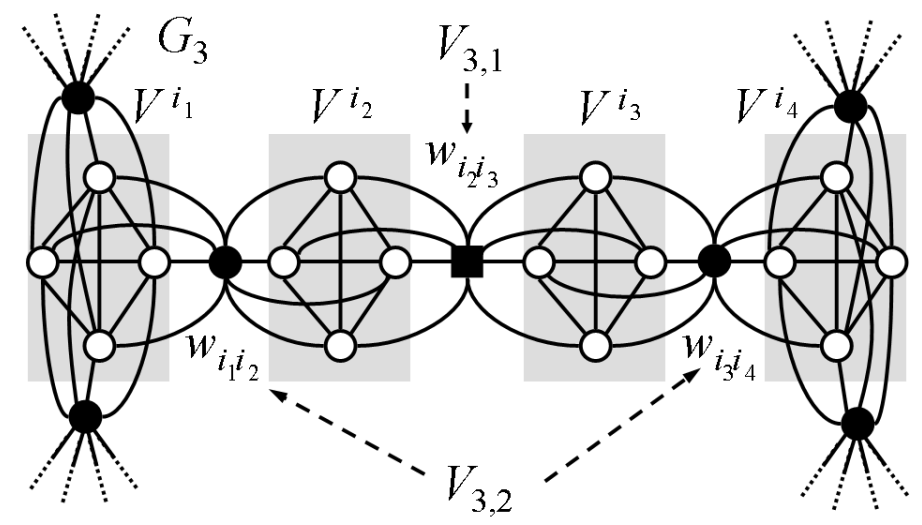

Fig. 3. Illustration of a subgraph of $G_{3}$ in the proof of Lemma 19 constructed from $G_{2}$, where $\left\{w_{i_{2}}, w_{i_{3}}\right\} \subseteq V_{2, E},\left\{w_{i_{1}}, w_{i_{4}}\right\} \subseteq V_{2}-V_{2, E}$, and $\left\{\left(w_{i_{1}}, w_{i_{2}}\right),\left(w_{i_{2}}, w_{i_{3}}\right),\left(w_{i_{3}}, w_{i_{4}}\right)\right\} \subseteq E_{2}$. Each vertex in $V_{3,1}$ and $V_{3,2}$ is drawn as a black square and a black circle, respectively.

For each $w_{i} \in V_{2}$, we construct the complete graph $\left(V^{i}, E^{i}\right)$ with $\left|V^{i}\right|=4$. For each $e=\left(w_{i}, w_{j}\right) \in E_{2}$, we construct one vertex $w_{i j}$. Let $V_{3,1}=\left\{w_{i j} \mid\right.$ $\left.\left(w_{i}, w_{j}\right) \in E_{2}, i<j,\left\{w_{i}, w_{j}\right\} \subseteq V_{2, E}\right\}$ and $V_{3,2}=\left\{w_{i j} \mid\left(w_{i}, w_{j}\right) \in E_{2}\right.$, $\left.w_{i} \in V_{2, E}, w_{j} \in V_{2}-V_{2, E}\right\}$. We construct $G_{3}$ from $G_{2}$ by replacing each vertex $w_{i} \in V_{2}$ by $\left(V^{i}, E^{i}\right)$ and each edge $e=\left(w_{j}, w_{\ell}\right) \in E_{2}$ by the vertex $w_{j \ell}$, and adding edges connecting $w_{j \ell}$ and $V^{j} \cup V^{\ell}$ for each edge $e=\left(w_{j}, w_{\ell}\right) \in E_{2}$; let 
$V_{3}=\left(\cup_{w_{i} \in V_{2}} V^{i}\right) \cup V_{3,1} \cup V_{3,2}$ and $E_{3}=\left(\cup_{w_{i} \in V_{2}} E^{i}\right) \cup\left(\cup_{w_{i j} \in V_{3,1} \cup V_{3,2}}\left\{\left(w_{i j}, u\right) \mid u \in\right.\right.$ $\left.V^{i} \cup V^{j}\right\}$ ) (see Fig. 3). Let $d(x)=3$ for each vertex $x \in V_{3,1}$ and $d(x)=4$ for each vertex $x \in V_{3,2}$ and $d(x)=0$ otherwise. Clearly, $G_{3}$ can be constructed in polynomial time in $n_{1}$ and $m_{1}$.

In [7], the following properties were shown.

Claim 20 (i) Let $X_{1}$ be a vertex cover in $G_{1}$. Then, $X_{2}=X_{1} \cup\left\{v_{i, j} \in V_{2, E} \mid\right.$ $\left.\left(v_{i}, v_{j}\right) \in E_{1}, v_{i} \notin X_{1}\right\} \cup\left\{v_{i, j} \in V_{2, E} \mid\left(v_{i}, v_{j}\right) \in E_{1}, i<j,\left\{v_{i}, v_{j}\right\} \subseteq X_{1}\right\}$ is a vertex cover in $G_{2}$. Moreover, the vertex set obtained by choosing exactly one vertex in $V^{i}$ for each $w_{i} \in X_{2}$ is a source set in $G_{3}$.

(ii) Let $S$ be a source set in $G_{3}$. Let $S^{\prime}$ be the vertex set obtained from $S$ by replacing each $w_{i j} \in\left(V_{3,1} \cup V_{3,2}\right) \cap S$ with some $w^{\prime} \in V^{i} \cup V^{j}$, and $X_{2}=\left\{w_{i} \in V_{2} \mid V^{i} \cap S^{\prime} \neq \emptyset\right\}$ be the vertex set in $G_{2}$. Let $X_{2}^{\prime}$ be the vertex set obtained from $X_{2}$ by replacing each $v_{i, j}$ with $\left\{v_{i, j}, v_{j, i}\right\} \subseteq V_{2, E} \cap X_{2}$ with $v_{i}$. Then $X_{2}^{\prime} \cap V_{1}$ is a vertex cover in $G_{1}$.

By this claim, we observe that $G_{1}$ has a vertex cover with cardinality at most $k$ if and only if $G_{3}$ has a source set with cardinality at most $k+m_{1} ; \operatorname{opt}\left(G_{3}, d\right)=$ opt $_{V C}\left(G_{1}\right)+m_{1}$ holds, where $\operatorname{opt}_{V C}(G)$ denotes the minimum size $|X|$ of a vertex cover $X$ in $G$. Now since $G_{1}$ is 3 -regular, we have $m_{1} \leq 3 o p t_{V C}\left(G_{1}\right)$. It follows that $\operatorname{opt}\left(G_{3}, d\right)=\operatorname{opt}_{V C}\left(G_{1}\right)+m_{1} \leq 4 o p t_{V C}\left(G_{1}\right)$.

Let $S$ be an arbitrary source set in $G_{3}$, and $X$ be a vertex cover in $G_{1}$ obtained from $S$ according to Claim 20(ii). Note that $|X| \leq|S|-m_{1}$. Then, we have

$$
\frac{|X|-\operatorname{opt}_{V C}\left(G_{1}\right)}{\operatorname{opt}_{V C}\left(G_{1}\right)} \leq 4 \frac{\left(|S|-m_{1}\right)-\left(\operatorname{opt}\left(G_{3}, d\right)-m_{1}\right)}{\operatorname{opt}\left(G_{3}, d\right)}=4 \frac{|S|-\operatorname{opt}\left(G_{3}, d\right)}{\operatorname{opt}\left(G_{3}, d\right)} .
$$

Therefore, if we would have a polynomial-time approximation scheme for 4LVSLP, then we would have a polynomial-time approximation scheme for VC3R.

\section{Concluding Remarks}

In this paper, given an undirected graph $G=(V, E)$ and a demand function $d: V \rightarrow Z_{+}$, we have considered the problem of finding a set $S \subseteq V$ with the minimum cardinality such that for every vertex $v$, there exist $d(v)$ paths between every vertex $v \in V-S$ and $S$ such that no pair of paths has a common vertex in $V-v$. We have shown that a simple greedy algorithm finds a $\max \left\{d^{*}, 2 d^{*}-6\right\}$-approximate solution to the problem in $O\left(\min \left\{d^{*}, \sqrt{n}\right\} d^{*} n^{2}\right)$ time. Especially, restricted to $d^{*} \leq 4$, we have given a tight analysis to show 
that it achieves an approximation ratio of 3, while the problem is APX-hard. However, it is still open whether the problem is approximable within a constant which is independent of $d^{*}$.

Acknowledgments: We are very grateful to the anonymous referees for careful reading and suggestions. This research was partially supported by the Scientific Grant-in-Aid from Ministry of Education, Culture, Sports, Science and Technology of Japan.

\section{References}

[1] P. Alimonti, V. Kann, Some APX-completeness results for cubic graphs, Theoretical Computer Science 237 (2000) 123-134.

[2] K. Arata, S. Iwata, K. Makino, S. Fujishige, Locating sources to meet flow demands in undirected networks, Journal of Algorithms 42 (2002) 54-68.

[3] M. Bárász, J. Becker, A. Frank, An algorithm for source location in directed graphs, Operations Research Letters 33 (3) (2005) 221-230.

[4] S. Even, R. E. Tarjan, Network flow and testing graph connectivity, SIAM Journal on Computing 4 (1975) 507-518.

[5] J. Heuvel, M. Johnson, Transversals of subtree hypergraphs and the source location in digraphs, CDAM Research Report LSE-CDAM-2004-10, London School of Economics (2004).

[6] T. Ishii, H. Fujita, H. Nagamochi, Minimum cost source location problem with local 3-vertex-connectivity requirements, Theoretical Computer Science 372 (1) (2007) 81-93.

[7] T. Ishii, H. Fujita, H. Nagamochi, Source location problem with local 3-vertexconnectivity requirements, Discrete Applied Mathematics 155 (2007) 25232538 .

[8] H. Ito, Telecommunication network simplifying problem - connectivity, area graph and T-mixed cut, NTT R\&D 44 (4) (1995) 367-372, (in Japanese).

[9] H. Ito, M. Ito, Y. Itatsu, K. Nakai, H. Uehara, M. Yokoyama, Source location problems considering vertex-connectivity and edge-connectivity simultaneously, Networks 40 (2) (2002) 63-70.

[10] H. Ito, K. Makino, K. Arata, S. Honami, Y. Itatsu, S. Fujishige, Source location problem with flow requirements in directed networks, Optimization Methods and Software 18 (2003) 427-435. 
[11] G. Kortsarz, Z. Nutov, A note on two source location problems, Journal of Discrete Algorithms 6 (2008) 520-525.

[12] M. Labbe, D. Peeters, J.-F. Thisse, Location on networks, in: M. O. B. et al. (ed.), Handbooks in Operations Research and Management Science, vol. 8, North Holland, Amsterdam, 1995, pp. 551-624.

[13] H. Nagamochi, T. Ibaraki, A linear-time algorithm for finding a sparse $k$ connected spanning subgraph of a $k$-connected graph, Algorithmica 7 (1992) $583-596$.

[14] H. Nagamochi, T. Ibaraki, Algorithmic Aspects of Graph Connectivity, Cambridge University Press, 2008.

[15] H. Nagamochi, T. Ishii, H. Ito, Minimum cost source location problem with vertex-connectivity requirements in digraphs, Information Processing Letters 80 (6) (2001) 287-294.

[16] M. Sakashita, K. Makino, S. Fujishige, Minimum cost source location problems with flow requirements, Algorithmica 50 (2008) 555-583.

[17] H. Tamura, M. Sengoku, S. Shinoda, T. Abe, Location problems on undirected flow networks, IEICE Transactions E73-E (12) (1990) 1989-1993.

[18] H. Tamura, H. Sugawara, M. Sengoku, S. Shinoda, Plural cover problem on undirected flow networks, IEICE Transactions J81-A (1998) 863-869, (in Japanese). 